may play a role, the cause of this association remains to be determined. The role of PEBD prior to PD warrants further evaluation in the context of a well-designed prospective clinical trial.

Competing interests None declared.

\section{PMO-218 COLONOSCOPY IN PATIENTS PRESENTING WITH MELAENA AND A NORMAL UPPER GASTROINTESTINAL ENDOSCOPY: A RETROSPECTIVE REVIEW FROM A SINGLE UK CENTRE}

doi:10.1136/gutjnl-2012-302514b.218

${ }^{1} \mathrm{E}$ Russo, ${ }^{*} \mathrm{~L}$ Hicks, ${ }^{2} \mathrm{~J}$ Hoare, ${ }^{2} \mathrm{H}$ Thomas, ${ }^{2} \mathrm{~J}$ Teare, ${ }^{2} \mathrm{H}$ Williams, ${ }^{2} \mathrm{~T}$ Orchard. ${ }^{1}$ Imperial College London, London, UK; ${ }^{2}$ Imperial College Healthcare NHS Trust, London, UK

Introduction Colonoscopy is frequently performed in patients presenting with melaena who have a negative upper gastrointestinal endoscopy (UGIE). Published literature suggests a diagnostic yield of $8 \%-30 \%$, the most common pathologies being colonic angiodysplasia and right-sided tumours. However these conditions often give rise to occult haemorrhage and a microcytic profile before patients present with overt bleeding. In patients presenting de novo with melaena a raised urea is known to be predictive of upper GI haemorrhage before any endoscopic assessment. Our aim was to examine the value of colonoscopy in the subgroup of patients with a negative UGIE, and to assess whether the absence of a raised blood urea and/or the presence of a microcytic erythrocyte profile at presentation are predictors of positive colonoscopy.

Methods Our reporting software was interrogated for the interval November 2007-October 2011. All cases of colonoscopy where melaena was the main indication, and which were preceded by a negative UGIE were analysed. In addition, we collected data on the admission blood urea and mean corpuscular volume (MCV). Patients for whom altered/fresh rectal bleeding were included in the indications in addition to melaena were excluded.

Results 724 patients had a total of 829 endoscopic evaluations of melena, and of these 62 patients (53\% female) with a median age of 69 year (range 27-91) met our inclusion criteria. 6 of 62 (9.6\%) had a cause for the melena identified on colonoscopy: cecal angiodysplasia in $2 / 6$, right-sided malignancies in $2 / 6$ and right-sided diverticular bleeds in $2 / 6$. The admission urea was not significantly lower in patients with a positive colonoscopy (median $11.5 \mathrm{mmol} / \mathrm{l}$, range $5.1-14.7$ ) compared to those with a negative colonoscopy (median $7.2 \mathrm{mmol} / \mathrm{l}$, range $1.4-33.6)(\mathrm{p}=0.43)$. Admission MCV however was significantly lower in patients with a colonic haemorrhage (median $77 \mathrm{fL}$, range 64-89) compared to patients with a negative colonoscopy (median $90 \mathrm{fL}$, range 66-116) $(\mathrm{p}=0.012$ ), with $3 / 6$ (50\%) having a low MCV compared to $5 / 56$ (8.9\%) of those with a negative colonoscopy (normal $=84-99 \mathrm{fL}$ ).

Conclusion The diagnostic yield of colonoscopy in patients with melaena and a non-contributory UGIE in our centre was low (9.6\%). A normal/low blood urea on admission did not predict a positive diagnosis for the haemorrhage at colonoscopy in our cohort. However, patients with a colonic source of bleeding had a significantly lower MCV, suggesting a chronic natural history for such right sided colonic haemorrhages.

Competing interests None declared.

\section{REFERENCES}

Tedesco FJ. Gastrointest Endosc 1981.

Ibach MB. Dig Dis Sci 1995.

3. Blatchford 0. Lancet 2000.
PM0-219 HIGHER THAN EXPECTED FALSE NEGATIVE CLO TEST IN PATIENTS NOT TAKING PPI ASSOCIATED WITH REGULAR ALCOHOL INTAKE AND ABSENCE OF ENDOSCOPIC GASTRITIS

doi:10.1136/gutjnl-2012-302514b.219

F Tahir, ${ }^{*}$ S Arshad, R C Evans, S Ashraf, H Elfaki, M Purcell-Jones. Department of Gastroenterology, Llandudno General Hospital, Llandudno, UK

Introduction $H$ pylori has a prevalence of around $40 \%-50 \%$ in the UK. ${ }^{1}$ Rapid urease test (CLO test) is commonly used in the endoscopy units around the UK to detect $H$ pylori. False negative CLO test results are associated with early reading of the test and use of acid suppressing medication. ${ }^{2}$ We assessed the reliability of CLO test and prevalence of false negative results.

Methods Retrospective data analysis was performed by auditing case notes of 85 patients with positive histology for $H$ pylori. CLO test was performed by using Kimberly-Clarke CLO test kit and the reading time was between 12 and $24 \mathrm{~h}$.

Results Male:Female ratio was 43:42. False negative CLO test was found in 37 patients (43.6\%), out of which 21 (56.7\%) were not taking PPI or stopped PPI for at least 2 weeks prior to the endoscopy. $16(43.2 \%)$ patients in the false negative group were drinking alcohol regularly as compared to $11(22.9 \%)$ in CLO positive group, while 28 $(58.3 \%)$ in CLO positive group were non drinkers. Regular drinkers taking PPI before the test had a low percentage $(3 / 12 ; 25 \%)$ of CLO positive results while non-drinkers not on PPI show a high percentage $(28 / 36 ; 77.8 \%)$ for positive results $[p=0.013]$. Also, in patients who had false negative CLO test, approximately $45 \%$ of patients consume regular alcohol. Absence of gastritis was associated with a slightly higher rate of false negative CLO test result ( $27.1 \%$ vs $20.8 \%$ for CLO positive) $[p=N S]$. Use of PPI only showed to contribute to false negative CLO test in absence of gastritis endoscopically $(70 \%$ in patients with no gastritis and taking PPI) $[p=N S]$. Smoking was not associated with false negative CLO test.

Conclusion High incidence of false negative CLO test result in our study suggests that CLO alone might not be a reliable test even in patients not taking acid suppressing medication. Regular alcohol use may contribute to false negative CLO test results. Gastric histology is better than CLO test in patients who are regular alcohol drinkers and taking PPI but in whom OGD does not show gastritis. Further studies need to be done to consider role of targeted gastric biopsies to increase the yield of CLO test.

Competing interests None declared.

\section{REFERENCES}

1. Fuccio $\mathbf{L}$, Laterza $\mathrm{L}$, Zagari $\mathrm{RM}$, et al. Treatment of $H$. pylori infection. $B M J$ 2008; 15;337.

2. Prince MI, et al. The CLO test in the UK, inappropriate reading and missed results Eur J Gastroenterol Hepatol 1999.

\section{PMO-220 PATIENT ANTICIPATION OF SOME PAIN GOES ALONG WAY WHEN PREDICTING OVERALL SATISFACTION WITH A COLONOSCOPY PROCEDURE}

doi:10.1136/gutjnl-2012-302514b.220

F-U-R Ali. ${ }^{*}$ Gastroenterology, Walsall Manor Hospital, Walsall, UK

Introduction Providing a quality patient experience is a key facet of the Global Rating Score (GRS). Patient surveys are considered an integral means of assessing satisfaction. Meeting the patient's expectations is likely to influence their assessment of the procedure. 
Methods We examined the factors that might potentially influence patient satisfaction with their colonoscopic procedure using a pre test questionnaire [self reported apprehension, the reason for any concerns, expectations of pain (represented as a visual analogue scale of $0-10,0=$ no pain and $10=$ maximum pain) and previous experience of colonoscopy]. Data collected during the test itself (patient self reported pain scores collected immediately post procedure and sedation doses used) were compared with a post test questionnaire of overall satisfaction and willingness to undergo the test again in the future if required.

Results 448 patients participated (287 females and 201 males). Age range 18-88years and mean age was 58 years. The mean anticipation of pain on the visual analogue scale was 2.61 . The most common causes of anxiety were "fear of cancer?" $(n=70)$ followed by "pain" ( $\mathrm{n}=35)$, "tear/perforation" $(\mathrm{n}=14)$ and "previous adverse endoscopic experiences" $(\mathrm{n}=9)$. The mean actual patient reported pain scores were 3.14. 63 patients (43 females and 19 males) was very worried before test and their average anticipated pain score (AtPS) was 4.19 (total average 2.61) and the actual pain score (AcPS) 3.48 (average 3.14). 225 patients were worried before test and their AtPS 2.95 and AcPS 3.37. The patients who were not worried, their AtPS were 1.74 and AcPS 2.8. Patients $(n=32)$ whose expected pre test pain scores were between 7 and 10 on visual analogue scale expressed higher levels of satisfaction with their procedures than those with lower anticipated pain scores (0-6) [93.7\% vs 73.8\%]. Patients who had a pre test apprehension score $>7$ were more agreeable to undergo the test again than those with score $<6$.

Conclusion Patient satisfaction is strongly correlated with patient comfort. Patients' appreciating that colonoscopy is a potentially painful procedure report a higher level of satisfaction and acceptance of the sedation offered. The importance appropriate preparation of the patient should not be underestimated.

Competing interests None declared.

\section{REFERENCE}

1. Global Rating Scale.

\section{Abstract PM0-220 Table 1}

\begin{tabular}{|c|c|c|c|c|}
\hline Groups & $\begin{array}{l}\text { Total } \\
\text { number, } \\
\mathbf{4 8 8}\end{array}$ & $\begin{array}{l}\text { Anticipated } \\
\text { pain score } \\
\text { (A 2.61) }\end{array}$ & $\begin{array}{l}\text { Actual } \\
\text { pain score } \\
\text { (A 3.14) }\end{array}$ & $\begin{array}{l}\text { Pt satisfaction } \\
a-c(a=\text { very, } \mathbf{c}=\text { none }) \%\end{array}$ \\
\hline \multirow[t]{3}{*}{ (A) Very worried } & 63 & 4.19 & 3.48 & $a=87$ \\
\hline & & & & $b=8$ \\
\hline & & & & $c=5$ \\
\hline \multirow[t]{3}{*}{ (B) Worried } & 225 & 2.95 & 3.37 & $a=82$ \\
\hline & & & & $b=14$ \\
\hline & & & & $c=4$ \\
\hline \multirow[t]{3}{*}{ (C) Not worried } & 200 & 1.74 & 2.8 & $a=78$ \\
\hline & & & & $\mathrm{b}=18$ \\
\hline & & & & $c=4$ \\
\hline
\end{tabular}

\section{PM0-221 APPROPRIATENESS OF FOLLOW-UP INDICATIONS AFTER COLONIC POLYP REMOVAL: AUDIT OF CURRENT PRACTICE IN A DISTRICT GENERAL HOSPITAL}

doi:10.1136/gutjnl-2012-302514b.221

J K J Diss, A Hashim, D Arokianathan, J Colquhoun, G Tritto.* Department of Gastroenterology, King George Hospital, Goodmayes, UK

Introduction Demand for colonoscopy is increasing worldwide due to the implementation of colon cancer screening programmes. According to current guidelines, the indication and timing for surveillance colonoscopy after removal of colonic adenomas are based on a risk estimation taking into account number and size of adenomas removed. In this study we audited the compliance with guidelines in the current practice of two district general hospital endoscopy services.

Methods The study was conducted at the King George and Queen's Hospitals in Essex (UK). All colonoscopies from June to August 2011 were retrospectively reviewed. All polyp-finding colonoscopies were selected and relevant data were retrieved from electronic records, patient notes and histopathology reports. The appropriate follow-up indication was established according to current British Society of Gastroenterology (BSG) guidelines, taking into account previous risk status, number and size of colonic adenomas. Finally the ideal indication was compared with the actual follow-up indication given to each patient.

Results A total of 1438 colonoscopies were reviewed. Polyps were found and removed in 314 (22\%). Only 205 were included in further analysis. The remaining 109 were excluded because further followup indication was based on different issues (12 IBD, 19 colon cancers, 34 technical problems, 21 unknown previous risk status, 23 referred for surgery). Of the included 205, 34 patients were given an appointment in 1 year (high risk), one in 2 years, 28 in 3 years (intermediate risk), 142 in 5 years or no follow-up (low risk). The follow-up indication was compliant with BSG guidelines in 136 $(66.3 \%)$ cases. In $33(16.1 \%)$ patients the follow-up appointment was scheduled too early and in $4(2 \%)$ too late. The remaining 32 $(15.6 \%)$ were booked for later decision in outpatient clinic. They belonged to the intermediate $(\mathrm{No} .=28)$ and high $(\mathrm{No}=4)$ risk groups. Overall, in the 3-months period, 24 inappropriate colonoscopies and 32 extra outpatient appointments were scheduled for the following year alone. In a further step we reviewed 154 histopathology results of benign polyps and found that 22 (14.3\%) more patients could have had their follow-up appointment modified on the basis of the final histopathology finding (2 earlier and 20 later). Conclusion The overall compliance with BSG guidelines in the evaluated period was $66.3 \%$ which is higher to that reported in previous studies. Nevertheless in only 3 months a significant inappropriate workload for endoscopy and outpatient clinics was generated. A more careful compliance with guidelines and a review of histopathology results could save a significant number of unnecessary colonoscopies and outpatient appointments.

Competing interests None declared.

\section{PM0-222 PRE-OPERATIVE ENDOSCOPY IN BARIATRIC SURGERY PATIENTS}

doi:10.1136/gutjnl-2012-302514b.222

${ }^{1} \mathrm{G}$ Bahra, ${ }^{*} \mathrm{~A}$ Desai, ${ }^{2} \mathrm{~A}$ Thillainayagam, ${ }^{3} \mathrm{~A}$ Ahmed. ${ }^{1}$ Foundation Year Doctor, Charing Cross Hospital, London, UK; ${ }^{2}$ Gastroenterology, Charing Cross Hospital, London, UK; ${ }^{3}$ Bariatric Surgery, Charing Cross Hospital, London, UK

Introduction Some authors suggest the routine use of endoscopy in patients undergoing bariatric surgery in order to detect asymptomatic hiatal hernias, oesophagitis, and gastric ulcers. Our unit uses selective endoscopy. The current study analyses the indications and findings of upper GI endoscopy in pre-operative bariatric surgery patients.

Methods A retrospective analysis of all bariatric surgery patients referred for Upper GI endoscopy at Charing Cross Hospital from 1 January 2009 to 30 October 2011. During this time period, 1093 bariatric surgery cases were performed. These consisted of 542 laparoscopic gastric bypasses, 220 laparoscopic gastric band insertions, 223 laparoscopic sleeve gastrectomies and 108 revisional bariatric procedures. The Endoscopy units' electronic database of oesophagogastroduodenoscopies (OGDs) performed in that time period was analysed to determine how many bariatric surgery 